\title{
Tuberkulose - Geschichtliche Entwicklung, Status quo und Ausblick
}

\section{Tuberculosis - Historical Development, Current Status, Future Prospects}

Autoren

Institute
R. Loddenkemper ${ }^{1}$, R. Diel ${ }^{2}$, T. Schaberg ${ }^{3}$

Deutsches Zentralkomitee zur Bekämpfung der Tuberkulose, Berlin

Klinik für Pneumologie, Medizinische Hochschule Hannover

Zentrum für Pneumologie, Diakoniekrankenhaus Rotenburg (Wümme) gGmbH, Rotenburg an der Wümme eingereicht 22.6.2010

akzeptiert 22.6.2010

\section{Bibliografie}

DOI http://dx.doi.org/ 10.1055/s-0030-1255623 Pneumologie 2010; 64: 567-572 @ Georg Thieme Verlag KG Stuttgart · New York ISSN 0934-8387

\section{Korrespondenzadresse \\ Prof. Dr. Dr. h.c. Robert Loddenkemper DZK}

Stralauer Platz 34

10243 Berlin

rloddenkemper@

dzk-tuberkulose.de

\section{Zusammenfassung \\ $\nabla$}

Die Tuberkulose (TB) ist in der Menschheitsgeschichte eine der führenden, wenn nicht sogar die führende Todesursache. Weltweit gehört sie auch heute noch zu den zehn häufigsten Todesursachen und zählt zu den global brennendsten Public-Health-Problemen. In Deutschland wie in fast allen Industrieländern nehmen Inzidenz und Mortalität zwar kontinuierlich ab, global hat sich die Situation infolge der HIV-Koinfektion und der Zunahme mehrfach resistenter Erreger in den letzten Jahren aber dramatisch verschlechtert. Nur ein rasches und international konzertiertes Handeln kombiniert mit intensiven Forschungsanstrengungen, um die gegenwärtige Diagnostik, Therapie und Prävention der TB entscheidend zu verbessern, wird vor einer Entwicklung bewahren, die selbst im 21. Jahrhundert nicht mehr oder nur äußerst schwer in den Griff zu bekommen ist.

\section{Epidemiologie}

$\nabla$

Seit Jahrtausenden ist die TB als Infektionskrankheit des Menschen bekannt. Frühere Bezeichnungen waren „Phthisis“ (Schwindsucht) - daher „Phthisiologie“ für Tuberkulose-Heilkunde oder „Weiße Pest“. Archäologische Skelettfunde belegen ihr Vorkommen schon in der Steinzeit, später unter anderem auch im frühen Ägypten, im antiken Griechenland und im Römischen Reich. Vermutet wurde die infektiöse Übertragbarkeit schon länger, den endgültigen Nachweis führte aber erst Robert Koch 1882 mit der Entdeckung des Tuberkulose-Erregers, des Mycobacterium tuberculosis (M. tuberculosis).

In Preußen lag die Mortalität Ende des 19. Jahrhunderts bei 320/100000 Menschen, ein Siebtel der Bevölkerung starb an einer TB, in der arbeitsfähigen Bevölkerung sogar jeder Dritte. Die Zunahme der TB im 19. Jahrhundert war hauptsäch-

\section{Abstract \\ $\nabla$}

Tuberculosis is one of the leading causes, if not the leading cause of death in human history. Worldwide TB still belongs to the ten most frequent causes of death and represents one of the most urgent public health problems globally. In Germany as in almost all industrialised countries, the incidence and mortality has been continuously declining but globally the situation is worsening dramatically due to HIV coinfection and the increase of polyresistant TB strains. Only rapid and internationally concerted action with the cooperation of the affected countries and combined with intensified research efforts into new diagnostics, therapeutics, and vaccines will be able to prevent a development that will no longer be manageable even with $21^{\text {st }}$ century technology.

lich Folge der Industrialisierung, die zu Armut, Unterernährung, beengten Wohnverhältnissen und mangelnder Hygiene geführt hatte. Auch heute noch ist die TB vorwiegend eine Krankheit, die bevorzugt arme Menschen und besondere Risikogruppen befällt.

Kurz vor seinem Tod 1910 konnte Robert Koch berichten, dass die TB-Sterblichkeit sich in Preußen seit 1882 um rund $50 \%$ verringert hatte. Dieser Trend der kontinuierlichen Abnahme setzte sich bis heute fort, nur kurzfristig unterbrochen durch die Folgen der beiden Weltkriege ( $\bullet$ Abb. 1). 2008 wurden in Deutschland nur noch 4543 Neuerkrankungen (Inzidenz 5,5/100000) und 155 Todesfälle registriert. Zehn Jahre zuvor betrug die Zahl der Erkrankungsfälle noch 9974 $(\bullet$ Abb. 2).

2008 waren 44\% der Erkrankten im Ausland geboren, die Inzidenz war bei ausländischer Staatsangehörigkeit um den Faktor 5,4 höher als bei 


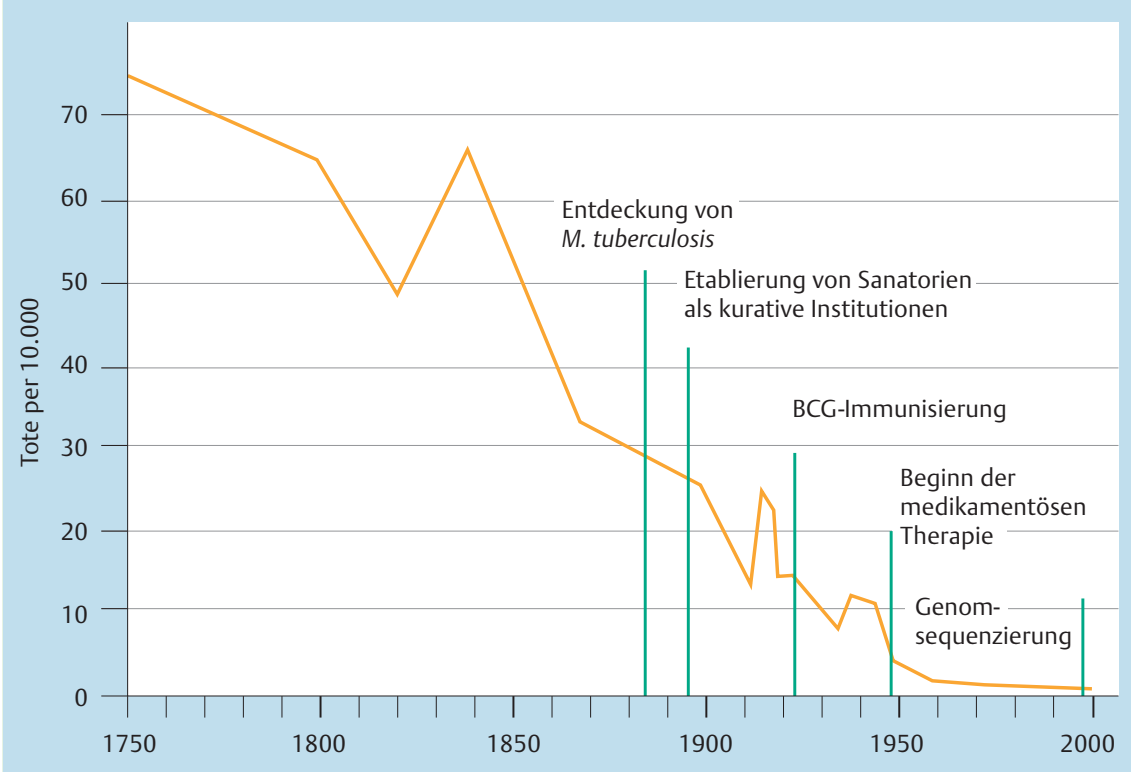

Abb. 1 Entwicklung der Tuberkulose-Mortalität in Deutschland seit 1750 und Zeitpunkte wichtiger diagnostischer, therapeutischer und präventiver Entwicklungen.

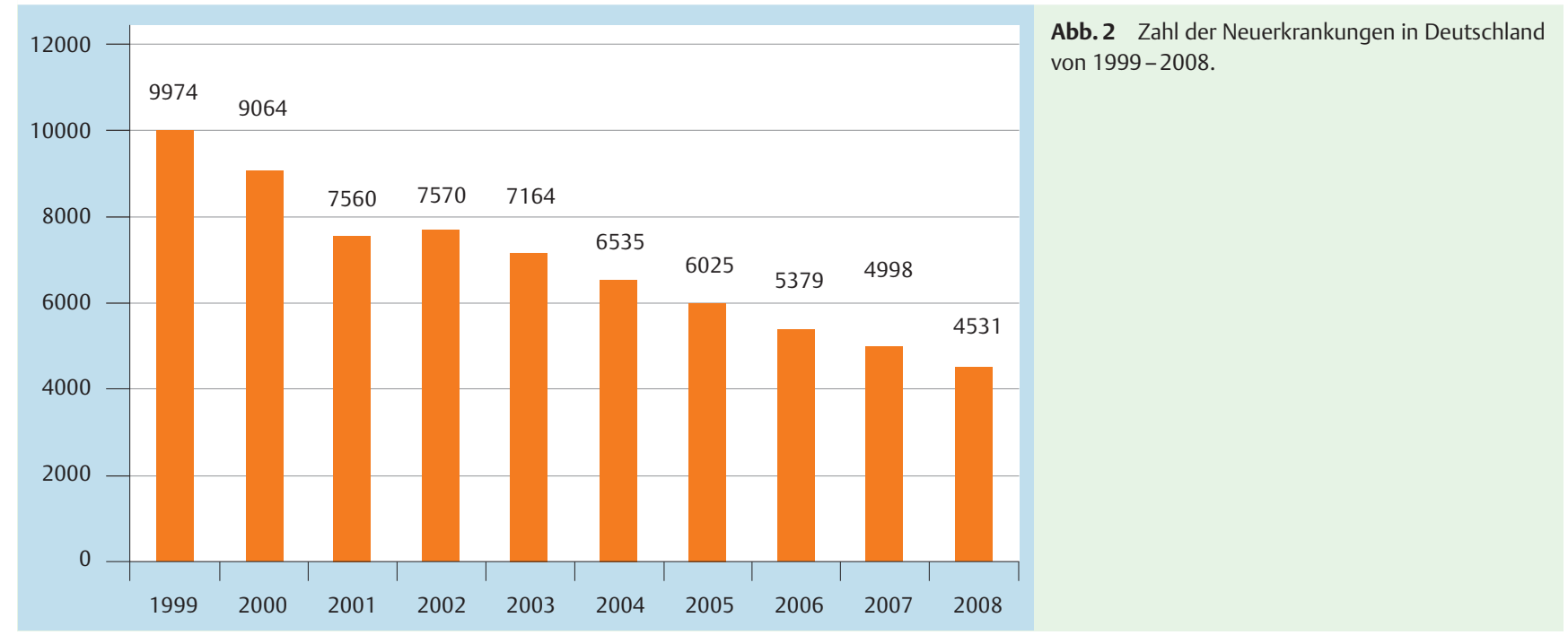

Deutschen. Eine Resistenz gegen die fünf wichtigsten Erstrangmedikamente lag in 11,6\% und eine Multiresistenz in 1,5\% der Fälle vor, vorwiegend bei Patienten aus der früheren Sowjetunion. Die Behandlung konnte in nur 81,5\% erfolgreich abgeschlossen werden, da in den höheren Altersklassen aufgrund der häufigen Multimorbidität ein Teil der Patienten vor Abschluss der Therapie an anderen Ursachen verstarb.

Weltweit schätzte die WHO die Zahl der Neuerkrankungen im Jahr 2008 auf 9,4 (8,9-9,9) Millionen und die der Todesfälle auf 1,8 Millionen, davon 500000 HIV-positiv. Am stärksten betroffen sind 22 „High-burden“-Länder, die 85\% aller Fälle ausmachen. In absoluten Zahlen führen Indien und China aufgrund ihrer großen Bevölkerung mit nahezu der Hälfte aller Fälle. Südafrika liegt mit einer Inzidenz über 900/100000 Population an der Spitze, die HIV-Prävalenz bei TB-Kranken beträgt dort bis zu 73\%, die Mortalität liegt bei 230/100000. Auch in einigen osteuropäischen Ländern steigen die HIV-Koinfektionsraten, häufig kombiniert mit einer MDR-TB.

2010 berichtet die WHO über einen unerwartet hohen Anstieg der Medikamenten-resistenten TB-Fälle. Mindestens 20\% sind zumindest gegenüber einem Erstrangmedikament unempfind- lich. Bei schätzungsweise einer halben Million Neuerkrankungen (ca. 5\% aller Fälle) liegt bereits eine Multi-Resistenz (MDR) vor, d.h. wenigstens gegen die beiden wichtigsten Medikamente Isoniazid (INH) und Rifampicin (RMP) ( $\bullet$ Abb. 3).

$50 \%$ dieser Fälle werden Indien und China zugeschrieben, der höchste Anteil an MDR unter neuen Fällen wurde mit 22,8$28,3 \%$ in einigen Regionen Russlands festgestellt. Allerdings werden global nur 7\% der geschätzten Fälle diagnostiziert und gemeldet, nur $1 \%$ wird nach internationalen Standards behandelt, nur ca. $60 \%$ werden geheilt, ein Drittel der Patienten stirbt. Geschätzte 25000 Fälle (5,4\% der MDR-Fälle) haben bereits eine extensive „Drug Resistance“ (XDR-TB), bei der neben der MDR zusätzlich eine Resistenz gegen eines der Fluorchinolone und eines der injizierbaren Antituberkulotika besteht ( $\bullet$ Abb. 4).

Katastrophal wirkt sich das Zusammentreffen von HIV-Koinfektion und XDR-TB aus, da in diesen Patientenkollektiven die Letalität exorbitant hoch ist. Einige Fälle haben sogar schon eine extrem resistente TB (XXDR-TB), bei der so gut wie keine Medikamente mehr wirken. Exakte Zahlen zur Resistenzsituation fehlen aber weltweit wegen unzureichender Laborkapazitäten, gerade in den am stärksten betroffenen Ländern. 
Anteil (\%) der MDR-Tuberkulose weltweit I Neue und vorbehandelte Fälle 2007

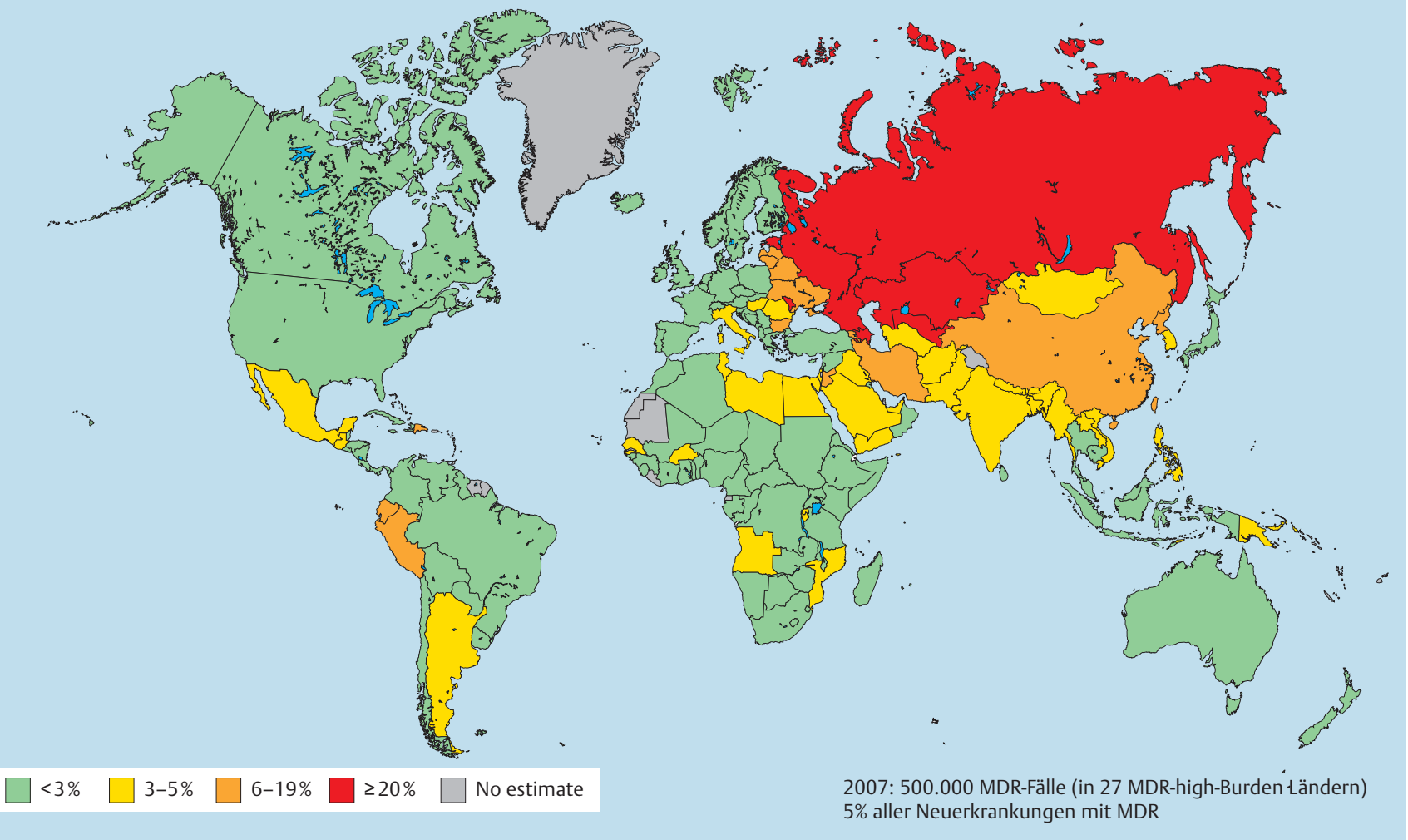

Abb. 3 Häufigkeit der MDR-Tuberkulose 2007 (neue und vorbehandelte Fälle) nach Ländern (WHO 2008).

\section{XDR-Tuberkulose}

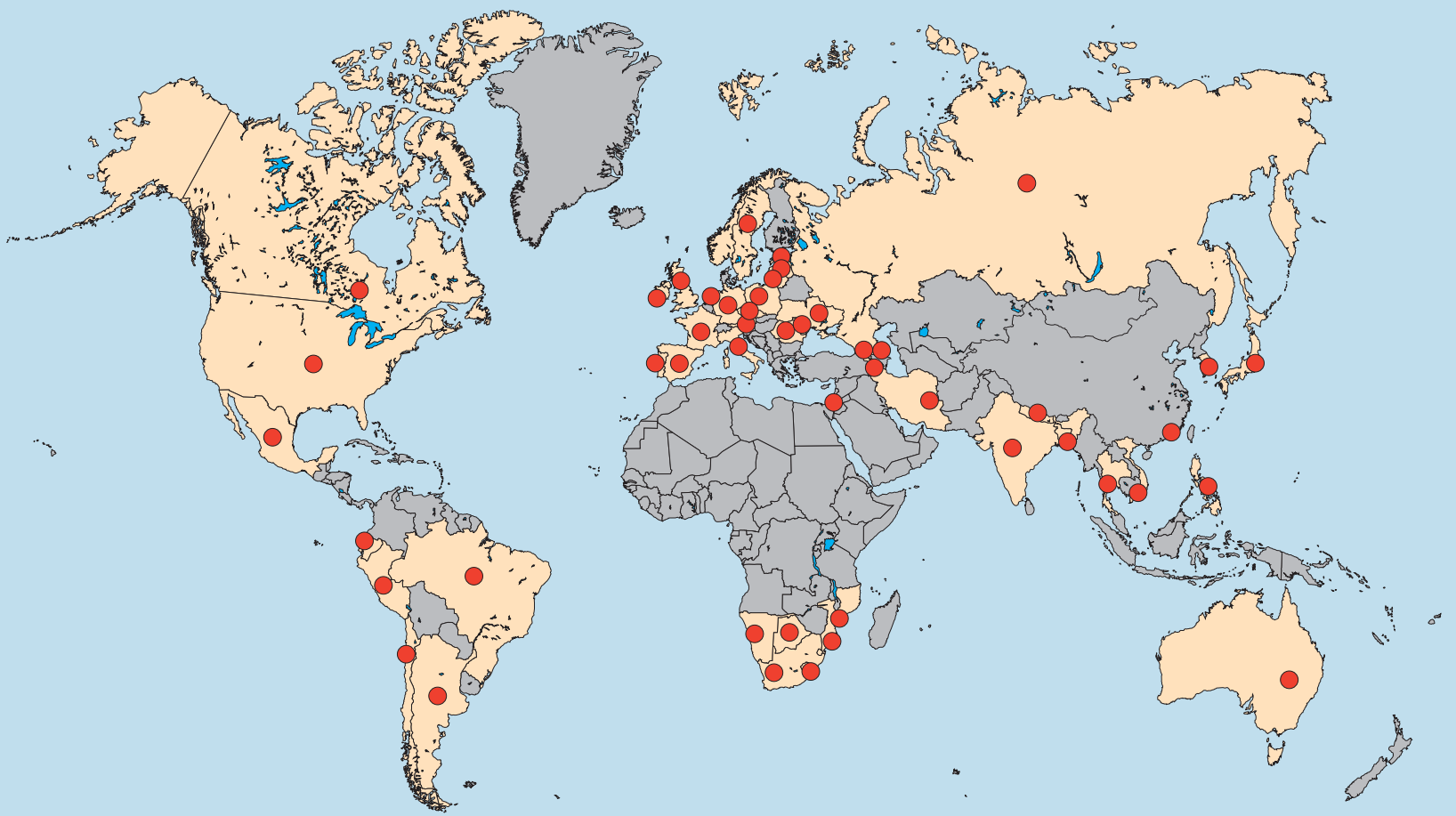

58 Länder haben mind. einen XDR-TB Fall gemeldet; Schätzwert: 25.000 neue XDR-Fälle jedes Jahr

Abb. 4 Länder, in denen bis Januar 2010 mindestens ein XDR-TB-Fall gemeldet worden ist (roter Punkt). 


\section{Diagnostik \\ $\nabla$}

Da das klinische Bild der TB häufig diagnostisch nicht richtungweisend ist, kommt bakteriologischen, bildgebenden und bioptischen Verfahren eine wesentliche Rolle in der Diagnostik zu.

Nach Robert Kochs Entdeckung des M. tuberculosis bestand die bakteriologische Diagnostik bis in die 1950er-Jahre vorwiegend im mikroskopischen Nachweis des Erregers mittels Ziehl-Neelsen-Färbung, obwohl er selbst schon Kulturen und Tierversuche angewandt hatte. Die direkte Sputum-Untersuchung ist zwar schnell und hoch spezifisch, hat aber nur eine niedrige Sensitivität. 1966 wurde die Fluoreszenzmikroskopie eingeführt, die fünffach schneller und auch spezifischer ist. Kulturen wurden zunächst fast ausschließlich für den Nachweis des Erregers in Geweben benutzt. Festmedien als Nährböden, wie die von Loewenstein-Jensen und andere, benötigen aber eine Wachstumsphase von 3-4 Wochen. Spezielle Flüssigmedien, die Anfang der 1970er-Jahre eingeführt wurden, erbringen den Nachweis bereits in der Hälfte der Zeit (1 - 2 Wochen) und mit höherer Sensitivität. Lange Zeit war das radiometrische Verfahren (BACTEC 460 TB) in Deutschland führend, inzwischen gibt es zahlreiche andere Flüssigmedien auf nicht-radioaktiver Basis, international am bekanntesten das MGIT-Verfahren (Becton Dickinson). Die Diagnose mittels Inokulation an Meerschweinchen, die besonders zwischen 1950 und 1970 populär war, ist inzwischen durch effizientere Kulturverfahren ersetzt. Empfindlichkeitsprüfungen gegenüber Medikamenten (Resistenztestungen) begannen in den 1950er-Jahren, als die Bedeutung der Resistenzentwicklung für die Therapie entdeckt wurde $($ Tab. 1).

Tab. 1 Fortschritte in der Diagnostik der Tuberkulose seit 1950.

\begin{tabular}{|ll}
\hline 1950 & Kultur (Löwenstein-Jensen Medium) \\
\hline $1950-1960$ & Medikamentenresistenztestung \\
\hline 1960 & Fluoreszenzmikroskopie \\
\hline 1977 & automatisierter radiometrischer Nachweis \\
1990 & genetische Marker („fingerprinting“) \\
1995 & DNA-Amplifikation (PCR) \\
1998 & Sequenzierung des kompletten Genoms von MTb \\
2000 & IFN-Gamma-Test für latente TB \\
2003 & schnelle Resistenztestung (GenoType MTBC) \\
\end{tabular}

Die Differenzierung der verschiedenen Mykobakterienarten heute spielen nicht-tuberkulöse Mykobakteriosen bei uns eine zunehmende Rolle - erfolgte zunächst mit konventionellen Verfahren wie Wachstumsmorphologie, typischen biochemischen Eigenschaften, Wachstum bei unterschiedlichen Temperaturen, Pigmentbildung und Anzüchtungszeiten, was aber mehrere Wochen in Anspruch nehmen kann. Heutzutage ist die Unterscheidung der verschiedenen Mykobakterienarten mittels Nukleinsäureamplifikations-Techniken (z. B. PCR) innerhalb von 2-3 Tagen möglich. Die Sensitivität der PCR für den Nachweis von Mykobakterien ist geringer als mit der Kultur, jedoch höher als mit der Mikroskopie.

Die im Jahr 1998 erfolgte Genom-Sequenzierung von M. tuberculosis ist ein bedeutender Meilenstein, der diagnostische Tests auf Gen-Basis ermöglicht. So wurden molekularbiologische Verfahren entwickelt, die mittels Gensonden eine raschere und genauere Artbestimmung ermöglichen und vor allem die Untersuchung auf Resistenzgene gegenüber einigen wichtigen Medikamenten erlauben, deren Ergebnisse bereits innerhalb 1 -2 Tagen vorliegen, sofern die Erreger direkt im Sputum nachweisbar sind. Beispiele hierfür sind der GenoType MTBDRplus Assay, der eine Re- sistenz gegenüber RMP und INH aufdeckt, die MTBDRsl-Version, die Resistenzen gegen Fluorochinolone, Aminoglykoside, Capreomycin und Ethambutol nachweist, sowie der Xpert MTB/RIF, der eine Resistenz gegen RMP aufdeckt.

Die Genom-Sequenzierung führte auch zu den Interferon-Gamma-Release-Assays (IGRAs), die akkurater im Nachweis einer latenten TB-Infektion (LTBI) als der Tuberkulin-Hauttest sind und auch bessere Vorhersagen zur Progression einer LTBI zu einer aktiven TB, aber nicht eine Differenzierung zwischen diesen beiden Formen erlauben.

Die 2003 von der WHO gegründete „Foundation of Innovative Drugs“ (FIND), zu deren Partnern zum Beispiel die supranationalen Referenzzentren für Mykobakterien in Borstel und Gauting zählen, hat sich zum Ziel gesetzt, genauere, einfachere, robustere und schnellere diagnostische Methoden zu entwickeln, die auch in den ärmeren Regionen der Welt an einer Vielzahl von Patienten angewandt werden können (www.finddiagnostic.org). Bis heute gilt aber das Paradox, dass moderne Entwicklungen, nicht nur in der Diagnostik, gerade den am meisten betroffenen Ländern nicht zur Verfügung stehen.

Die bildgebende Diagnostik wurde durch Wilhelm Conrad Röntgen 1895 eingeleitet und ist ein wesentlicher Pfeiler der TB-Diagnostik in den Industrieländern geworden. Anfang des vorigen Jahrhunderts wurde die Durchleuchtung bevorzugt, später wurden bei Röntgenreihenuntersuchungen Schirmbilder benutzt. Heute steht die konventionelle Röntgenaufnahme des Thorax im Vordergrund, ergänzt durch die Computertomografie und ggf. auch durch eine Positronen-Emissions-Tomografie-CT. Die flexible Bronchoskopie hat die Magensaftuntersuchung bei Verdacht auf eine pulmonale Tuberkulose, die sich im Sputum nicht nachweisen lässt, ersetzt, andere bioptische Verfahren werden insbesondere bei extrapulmonalen Tuberkulosen angewandt. Bioptisch gewonnenes Gewebe sollte nicht nur histologisch, sondern auch kulturell untersucht werden, nachträglich sind am fixierten Material auch Untersuchungen mittels PCR möglich.

\section{Therapie}

$\nabla$

Vor Einführung der Antibiotika-Therapie wurden verschiedenste Metallsalze und andere Substanzen in der Behandlung der TB versucht. Nach Scheitern der Tuberkulin-Therapie wurden Liegekuren in speziellen Lungenheilanstalten populär, die später ergänzt wurden durch die Kollapstherapie mit artifiziellem Pneumothorax und durch chirurgische Verfahren wie Phrenikotomie, extrapleurale Plombierung, Thorakoplastik und Lungenresektionen. Diese Behandlungen wurden aber in den 1950er-Jahren durch die Einführung der Antituberkulotika abgelöst, Streptomycin war 1944 das erste Antibiotikum in der TB-Therapie. Rasch stellte sich aber das Problem der Resistenzentwicklung aufgrund der Spontanmutationen von M. tuberculosis. Durch die Studien des British Medical Research Councils wurde die Überlegenheit einer Kombinationstherapie mit INH und PAS deutlich, was aber immer noch eine Behandlungszeit von zwei Jahren erforderte. 1960 ließ sich diese Behandlungsdauer durch das Ersetzen von PAS durch Ethambutol auf 18 Monate reduzieren, 1970 durch zusätzliche Gabe von Rifampicin auf 9 Monate und schließlich 1980 mittels Hinzufügen von Pyrazinamid auf 6 Monate ( $\bullet$ Abb.5).

Anzustreben ist aber eine noch kürzere Behandlungszeit, ein erster Schritt mag die Ergänzung mit einem Fluorochinolon sein; entsprechende Studien werden derzeit mit Moxifloxacin durchgeführt. 


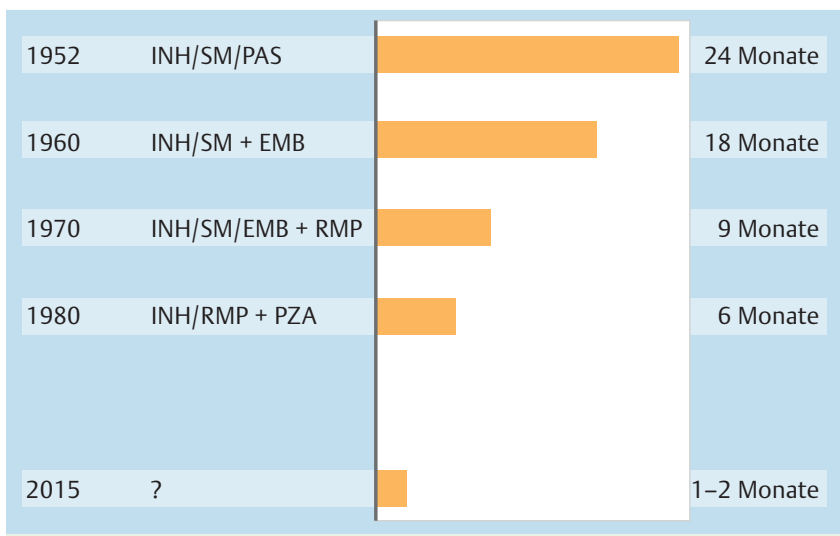

Abb. 5 Verkürzung der Standardtherapie-Dauer der Tuberkulose seit 1952 (INH = Isoniazid; SM = Streptomycin; PAS = Paraaminosalicylsäure; $\mathrm{EMB}=$ Ethambutol; RMP = Rifampicin; PZA = Pyrazinamid $)$.

Das mangelnde Interesse der Pharmaindustrie, neue Antituberkulotika zu entwickeln, ist durch die hohen Kosten von ca. 800 Millionen Dollar pro Medikament und den kaum vorhandenen Markt in den Industrieländern zu erklären.

Insbesondere wegen zunehmender Medikamentenresistenzen ist die Entwicklung neuer Medikamente jedoch dringend notwendig. Im Jahr 2000 wurde die „TB Alliance“ als eine öffentlichprivate Partnerschaft ins Leben gerufen. Derzeit gibt es eine Reihe von neuen Ansätzen zur medikamentösen Therapie, die zum großen Teil aber noch in der präklinischen Phase stecken (www. tballiance.org/newportfolio.php).

Die von der WHO 1991 eingeführte „DOTS“-Strategie (Directly Observed Treatment, Short Course) beinhaltet neben dem politischen Willen vonseiten der Regierungen der betroffenen Länder die passive Fallfindung mittels Untersuchung von Sputum-Ausstrichen (mindestens 70\% der Fälle sollen so entdeckt werden), die standardisierte Behandlung unter Einhaltung einer direkt überwachten Medikamenteneinnahme (erfolgreiche Behandlung in mindestens $85 \%$ der Fälle), wobei die Versorgung mit Medikamenten garantiert sein muss, sowie die Registrierung und Meldung aller Patienten. Dieses Programm wurde inzwischen durch „DOTS-Plus“ zur Behandlung der medikamentenresistenten Tuberkulosefälle ergänzt, wobei das Green Light Committee der WHO die Bereitstellung kostengünstiger Medikamente mit Hilfe des Global Fonds nur bei Vorliegen eines funktionierenden nationalen Tuberkulosekontrollprogramms befürwortet.

Ein wesentlicher Schwachpunkt ist derzeit die fehlende Laborkapazität zur Bestimmung der Resistenzen, sodass die Gefahr der Zunahme von MDR-, XDR- und XXDR-Tuberkulosen durch eine ungezielte Medikamenteneinnahme zunimmt.

Die Behandlung der MDR- und XDR-TB ist wegen der multiplen Nebenwirkungen und Interaktionen schwierig. Da die Wirksamkeit der zur Verfügung stehenden Medikamente außerdem deutlich geringer ist als bei den oralen Erstrang-Medikamenten, ist die Dauer der Therapie auch wesentlich länger und beträgt bis zu zwei Jahre nach der kulturellen Konversion. Die Heilungsraten bei MDR-TB liegen nur bei etwa $60 \%$, bei XDR-TB sogar noch darunter. Die Behandlungskosten sind um ein Vielfaches höher als bei medikamentensensibler TB.

In Einzelfällen wird bei polyresistenter TB auch die zusätzliche operative Resektion der befallenen Lungenanteile durchgeführt. Die Indikation ist insbesondere gegeben, wenn nicht ausreichend Medikamente zur Verfügung stehen, sowie bei ausbleibender kultureller Konversion, persistierenden Kavernen, weitgehend lokalisiertem Befall und fehlenden funktionellen Kontraindikationen.

Die unterstützende Rolle von immunmodulatorischen Medikamenten wie Interferone oder von Vitamin D ist dagegen noch unklar. Besonderheiten der Therapie sind bei HIV-Koinfektionen zu berücksichtigen. Heute geht der Trend dahin, möglichst früh eine antiretrovirale Therapie zusätzlich zur antituberkulotischen Behandlung einzuleiten, wobei aber auf das „Immune Reconstitution Inflammatory Syndrome“ (IRIS) zu achten ist.

Die WHO hat im Jahr 2006 einen ehrgeizigen globalen Plan (Millenium Development Goal) mit dem Ziel vorgelegt, bis zum Jahr 2015 im Vergleich zu 1990 die TB-Neuerkrankungsrate und die Zahl der Todesfälle um 50\% zu senken. In einigen Regionen der Welt scheint dieses Ziel erreicht zu werden, aber insbesondere in den afrikanischen Ländern südlich der Sahara und in den Ländern der früheren Sowjetunion stehen die Chancen dafür schlecht.

\section{Prävention \\ $\nabla$}

In der Prävention der TB standen seit dem Ausgang des 19. Jahrhunderts die Isolierung der ansteckenden Fälle sowie die Verbesserung der Lebensverhältnisse im Vordergrund. Die Heilstättenbewegung half bei der Isolierung, jedoch wurde bald die besondere Bedeutung der Tuberkulosefürsorge erkannt, einer Idee, die von Schottland ausging und zunächst besonders in Frankreich und Belgien als „Dispensaire“-Bewegung Verbreitung fand. In Deutschland kam es nur zögerlich zu einem flächendeckenden Aufbau des Fürsorgewesens, die erste Tuberkulosefürsorgestelle wurde 1899 in Halle errichtet. Zu ihren wesentlichen Aufgaben gehörten hygienische und finanzielle Hilfen für die Patienten und ihre Angehörigen sowie die Aufklärung über die Krankheit. Weitere Präventionsmaßnahmen waren später die aktive Fallfindung mittels Röntgenreihenuntersuchungen und die Ausrottung der bovinen TB durch Tötung infizierter Rinder in den 1950erJahren und durch die Pateurisierung der Milch.

1921 wurde von den Franzosen Calmette und Guérin die nach ihnen benannte BCG-Impfung eingeführt, deren Wirksamkeit sich aber vorwiegend auf die Verhinderung der tuberkulösen Meningitis und der Miliartuberkulose bei Kindern beschränkt. In Deutschland litt die Akzeptanz des Impfverfahrens unter dem „Lübecker Unglück“, als 1929/30 versehentlich lebende, nicht attenuierte Bakterien verabreicht wurden und 77 von 251 geimpften Kindern starben. In Deutschland wird die Impfung seit 1998 nicht mehr empfohlen, da die eingeschränkte Wirksamkeit die Impfkomplikationen nicht aufwiegen kann. In der früheren DDR wurde die routinemäßige Impfung erst nach der Wiedervereinigung eingestellt. Weltweit ist sie aber die am häufigsten angewandte Impfung.

Heute wird von verschiedenen Forschergruppen versucht, neue, auch bei Erwachsenen wirksame TB-Impfstoffe zu entwickeln, die sowohl eine primäre Erkrankung als auch eine Reaktivierung einer latenten Tuberkulose-Infektion verhindern sollen. Auch hier wurde international eine Public-Private-Partnership in Form der „AERAS - Global TB Vaccine Foundation“ gegründet (www.aeras.org). Da die Prüfung der Wirksamkeit neuer Impfverfahren, die zum Teil schon in der klinischen Phase sind, durch eine epidemiologische Beobachtung nur äußerst zeitraubend festzustellen wäre, wird nach Biomarkern gefahndet, die Erfolge frühzeitiger anzeigen können. 
Ein wichtiger Meilenstein in der Prävention der TB war 1955 die Einführung der präventiven Chemotherapie, zunächst in den USA bei im Tuberkulintest positiven Kindern durch die Behandlung mit Isoniazid. Diese kann heute gezielter bei Risikopatienten aufgrund der höheren Sensitivität und Spezifität der IGRA-Teste vorgenommen werden.

Massenscreening-Verfahren mittels Röntgenreihenuntersuchungen oder Tuberkulin-Testungen sind heute weitgehend durch Umgebungsuntersuchungen von ansteckungsfähigen Patienten sowie die aktive Fallfindung bei Risikogruppen ersetzt. Die Einführung molekularer epidemiologischer Verfahren („Fingerprinting") hat sich hierbei gegenüber den konservativen Methoden als äußerst hilfreich erwiesen, wird aber erst in wenigen Ländern, auch nicht in Deutschland, flächendeckend genutzt. Mittels aktiver Fallsuche wurden 2008 in Deutschland insgesamt 435 Fälle (11,3\% aller 4543 Fälle) entdeckt, aufgrund einer Umgebungsuntersuchung 266 Fälle (7\%) - bei Kindern aber 56 von 115 Fällen (49\%) - und aufgrund der Untersuchungen von Risikogruppen 166 Fälle (4,3\%).

Ganz wesentlich ist in der Prävention die frühzeitige Erkennung einer ansteckungsfähigen TB und die rasche Einleitung einer resistenzgerechten Behandlung. Bei voll sensiblen Erregern führt die Therapie bereits nach $2-3$ Wochen zu einer klinisch relevanten Verminderung der Erreger-Ausscheidung im Sputum. Wichtig ist es, die Vorschriften zur Infektionsverhütung strikt zu beachten.

Das WHO-Ziel der globalen Eliminierung der TB bis zum Jahr 2050 (weniger als eine Tuberkulose-Neuerkrankung auf eine Million Einwohner) erscheint zum jetzigen Zeitpunkt utopisch, es sei denn, dass neue Impfverfahren eine entscheidende Wende bringen.

\section{Interessenkonflikte}

$\nabla$

Die Autoren geben an, dass kein Interessenkonflikt besteht.

\section{Literatur}

1 Barry CE 3rd, Boshoff HI, Dartois V et al. The spectrum of latent tuberculosis: rethinking the biology and intervention strategies. Nature Reviews Microbiology 2009; 3: 845-855

2 Diel $R$, Loddenkemper R, Nienhaus A. Evidence-based comparison of commercial interferon-gamma release assays for detecting active TB: a metaanalysis. Chest 2010; 172: $952-968$

3 Dye C, Williams BG. The population dynamics and control of tuberculosis. Science 2010; 328: 856-861

4 Gandhi NR, Shah NS, Andrews JR et al. HIV coinfection in multidrug- and extensively drug-resistant tuberculosis results in high early mortality. Am J Respir Crit Care Med 2010; 181: 80-86

5 Kaufmann SHE, Hussey G, Lambert PH. New vaccines for tuberculosis. Lancet 2010; May 18 [Epub ahead of print]

6 Loddenkemper R, Hauer B. Resistente Tuberkulose. Große Herausforderung durch eine Weltepidemie. Dtsch Arztebl Int 2010; 107: 10-19

7 Mitchison DA. The diagnosis and therapy of tuberculosis during the past 100 years. Am J Respir Crit Care Med 2005; 171: 699- 706

8 Richter E, Rüsch-Gerdes S, Hillmann D. Drug-susceptibility testing in TB: current status and future prospects. Expert Rev Resp Med 2009; 3: 497-510

9 Robert Koch-Institut. Bericht zur Epidemiologie der Tuberkulose in Deutschland für 2008. Robert Koch-Institut, Berlin 2010

10 Russell DG, Barry CE 3rd, Flynn JL. Tuberculosis: what we don't know can, and does, hurt us. Science 2010; 328: 852 - 856 